\title{
¿VALORES EN UNA SOCIEDAD ADOLESCENTE? \\ RETOS DE UNA EDUCACIÓN DIFERENTE
}

Agustín de la HERRÁN ${ }^{1}$

RESUMEN: Atravesamos una crisis general, que algunos autores asocian a una inmadurez social generalizada. En ese contexto se deducen retos educativos como consecuencia de redefinir percepciones normalmente admitidas.

PALABRAS CLAVE: Inmadurez social. Egocentrismo. Educación de la conciencia.

\section{Un diagnóstico global}

A cien mil Km., un observador percibiría que la humanidad está fragmentada. A la misma distancia y con un telescopio electrónico, admitiría que el agente divisor no es exterior, porque está ligado a la práctica del egocentrismo desde la génesis y el desarrollo del “pensamiento egocéntrico” y a la correspondiente forma de actuar de personas, instituciones y colectivos, cuyas prioridades suelen ser ceñirse a su pequeño espacio de intereses y acciones y su continua preeminencia relativa. El impulso compensador, a saber, el anhelo de convergencia o de unidad es lábil, apenas ocupa lugar en la conciencia ordinaria. El fenómeno $\mathrm{y}$ el hecho es que componemos un todo con demasiadas particiones, grietas, muros, distancias. Un mundo cuajado de ismos que nunca llegarán a ser istmos, aunque se conecten por Internet. La Tierra sigue siendo como una escuela sin proyecto educativo: falla la motivación por lo ideal, que es una parte del conocimiento, y falta identificar ese ideal con el sentido universal. El resultado general es un gran deterioro externo e interno -desarrollo mal repartido e inmadurez social generalizada- en la sociedad del egocentrismo, que ha terminado con muchas vidas y con mucha humanidad. De hecho, esta muerte anticipada es lo que la impide nacer a su totalidad. Por esto: “Al mismo tiempo que vive sus pluralidades de vidas nacionales, la humanidad vive ya su muerte sin haber podido nacer todavía.” (MORIN, 1983, p.517).

La vida humana se estructura sobre un sinnúmero de sistemas más o menos amplios, cuyas metas primeras son sus propios intereses, rentabilidad y eficacia. En cada persona identificada suele predominar la “conciencia sistémica” de adoquín o de pieza del puzzle en que les ha tocado vivir. Los fragmentos son de toda índole, de todo contenido y de todo

\footnotetext{
${ }^{1}$ UAM - Universidad Autónoma de Madrid. Facultad de Formación de Profesorado y Educación .Departamento de Didáctica y Teoría de la Educación. Madrid - España. agustin.delaherran@uam.es
} 
color ${ }^{2}$. Este hecho social y personal define lo que hemos denominado “conciencia ordinaria”, y dibuja en el condicionamiento de personas y en la piel de las sociedades la ausencia de un norte común. ¡Y parece que casi todo el mundo está muy contento y hasta orgulloso, porque lo normal es considerar y valorar la propia partición (nacionalista, religiosa, política, cultural, deportiva, epistemológica...) como la más verdadera, importante o acertada! ¿Cómo podría ser de otra manera? La identificación normal con el propio sistema parcial tiene efectos que pasan por un fortalecimiento de una conciencia rentabilista, lo que asocia un apego a la eficacia, lo que precisa de una cierta insensibilidad hacia lo demás, lo que dificulta la autocrítica, lo que interfiere la posibilidad de rectificación, lo que impide percibir la autoimagen con ecuanimidad, lo que bloquea que se desarrollen sinapsis de generosidad, cooperación, convergencia y madurez, lo que evita la posibilidad de reidentificarse con unidades más amplias de un modo más generoso, situadas en una dimensión diferente o superior a "nuestra pequeña parcela de intereses”. Todos tienden a partir de que su posición y su sistema es el acertado, y sienten que son los demás quienes se encuentran en un error.

Todos defienden su postura de un modo formalmente semejante -es lo que denominamos “paradoja del ismo perfecto” (HERRÁN, 1997, p.75). Ahora bien, volvamos a la hipótesis del estadio preoperatorio generalizado: ¿ ¿se diferencia este comportamiento al de un conjunto de niños de cuatro años que corren detrás de una pelota, creyendo que los demás le acompañan, teniendo en cuenta que todos piensan lo mismo? Pudiendo ser así, ¿hay posibilidades educativas? No muchas, si no se ve o no se quiere reconocer el problema. En efecto, hasta tal punto pervive ese relativo conformismo o bienestar que, por ejemplo, resulta raro que nos preguntemos en qué medida esta ausencia de universalidad, como sentimiento y proyecto social, ha de preocupar o ha de atenderse en la planificación, el desarrollo y la evaluación de la enseñanza o la formación de los profesores, y en la conceptuación y uso de las TIC, con especial referencia a Internet. De hecho, alguien podría decir que la vida es así: limitada y para la limitación; que un interior polarizado a lo parcial es lo normal; que su desmembramiento está tan extendido que pasa desapercibido; que pensar en otra cosa es ingenuo, utópico o propio de chalados; que el discurso de la conciencia de marcos y metas más amplios no es lo concreto, que ni siquiera debería considerarse como contexto o como anhelo, porque va mucho más allá de lo controlable, etc. ¡Abultado error -diremos-. Pues es precisamente ese indiferentismo, esa negación lo que debería motivar que la educación

\footnotetext{
${ }^{2}$ Quizá en esto sí se ha avanzado: antes la realidad era en blanco y negro, ahora es en color. Dentro de un tiempo será holográmica, pero de seguir así no habrá cambiado esencialmente.
} 
reaccione. Y para nosotros es tan extraño que esto no ocurra como que un viajero que toma un avión no lo haga para desplazarse a algún destino.

Ahora bien, para este sentimiento de universalidad y anhelo de unidad, para la emergencia de una conciencia extraordinaria, para la imprescindible ausencia de estrechez de conciencia, ¿influye haber cursado el Bachillerato? ¿Pesa en algo ser universitario? ¿Los doctores ya han accedido a esto? ¿Y qué decir de los catedráticos de universidad? Pareciera, como se podrá advertir, que la limitación de la conciencia no está muy asociada a la altura de los grados académicos o a los desempeños profesionales. Las más altas cotas de preparación académica no se corresponden siempre con la formación para el desarrollo de la persona, ni siquiera de un modo especial en quienes están comprometidos con la formación de otros. Por tanto, nos preguntamos urgentemente: ¿Qué estamos dejando de hacer en educación y en formación?

Cambiemos la perspectiva: Quizá la educación que se está promoviendo, favoreciendo o desarrollando tenga algo que ver con esto. Las políticas educativas locales, etnocentristas, nacionalistas (secesionistas o centralistas) e internacionalistas son parciales por definición, y promueven una educación sesgada, si bien su diámetro puede ser mayor o menor. Ahora el referente no es nuestro Estado, sino su marco internacional cercano, según cada país: Europa, Latinoamérica, África... Pero en todos los casos su escora o parcialidad lo es por omisión de otras posibilidades más complejas, por ejemplo, Educar para la Universalidad. Del mismo modo, de una forma irrefutable, podemos asegurar que la operación "sustracción” es inferior lógicamente a la operación “cociente”. Pero el problema que abordamos no es cuestión sólo ni sobre todo de políticas educativas, sino de “pensamiento egocéntrico” generalizado. Ésta es nuestra tesis desde hace veinte años, y todavía nadie la ha refutado. Es carnaza para la indiferencia, otro rasgo del contexto interior de nuestros días. E pur si mueve.

\section{La inmadurez social generalizada, clave de la crisis social y educativa}

Inmadurez social generalizada: Parafraseando a A. Einstein -que se refirió a las dos primeras-, decimos que hay tres constantes universales: la velocidad de la luz, el egocentrismo (asiento de inmadurez o de estupidez) y el anhelo de conciencia. Pues bien, la velocidad de la luz no es objeto de estudio de la Didáctica. La superación del egocentrismo, que hemos definido como problema número 1 de la educación (HERRÁN, 1997), es prácticamente un ámbito inédito para la formación. Y el anhelo, el único impulso conocido que contrarresta la entropía, está inédito. Hoy está en primer plano lo que J.L. Pinillos ha llamado "razón instrumental" (comunicación personal), el ocio y el lucro, un par de ramas del egocentrismo 
humano, que en la cultura euroamericana, aflora como rentabilidad y placer como consecuencia de la consecución de objetivos.

E. Rojas (2004, p.20) concreta que estamos en una "sociedad adolescente”: “Una sociedad como la nuestra, cada vez más adolescente, se caracteriza por la inmadurez colectiva, y sus mensajes son tan contrapuestos que resulta muy difícil reconciliarlos.” En otro lugar señala:

Hoy se ha producido en el mundo moderno una socialización de la inmadurez y nos encontramos con masas de población adulta convertida en adolescente. También los criterios de madurez están en un tono auténticamente adolescente y ésta es un poco la realidad verdaderamente negativa en que nos encontramos. (ROJAS, 1990, p.13).

Si el egocentrismo -característica básica de la inmadurez y de la inteligencia del niño de 2 a 7 años, aproximadamente- es la dificultad para darse cuenta de que hay otros puntos de vista diferentes al propio y de que alguno de ellos puede ser mejor que el nuestro, o no hemos superado el estadio preoperatorio, o el egocentrismo es la característica más llamativa del adulto. Se deduce de ello que quizá no estemos tanto en una sociedad adolescente, sino en una sociedad infantilizada (HERRÁN, 2003, 2008). Puesto que el infantilismo adulto (personal y colectivo) es uno de los grandes retos inconfesables de la Educación, puede que en un futuro se reconozca la especial relevancia de los profesionales de la Educación Infantil y Primaria para la educación de adultos.

\section{Nuevos retos educativos desde posibles errores perceptivos}

Es posible deducir y concretar nuevos retos y ángulos educativos, como consecuencia de redefinir percepciones normalmente admitidas, para abordar los problemas de la educación con mayor complejidad:

1) Quizá no haya que educar para el progreso: Antes de redefinir la enseñanza, es preciso redefinir la idea que del desarrollo social se tiene. Todo parece indicar que lo que vale para progresar no siempre sirve para evolucionar. El desarrollo depende del progreso, pero la evolución depende del sentido de la complejidad de la conciencia. La conciencia resulta del conocimiento y de la educación de la razón, y se traduce en un desarrollo más sensible, inteligente, creativo y autoconsciente. Insistimos en las preguntas cruciales: “¿Qué estamos haciendo?”, “¿qué estamos construyendo?” En el Shichisuo, antiguo texto chino (h. 1600), se dice: "En el mundo vegetal los tallos crecen hacia arriba, y las raíces lo hacen hacia abajo. Pero en ningún momento la planta deja de nutrirse y de repararse”. Si trasladamos esta 
imagen al desarrollo de la humanidad podremos reconocer que lo de nutrirse o crecer hacia arriba el ser humano no lo hace del todo mal; que elimina peor, y que eso de repararse, como exige autocrítica y madurez personal, es lo que peor realiza. "Educar para el progreso” es una contradicción. "Educar para la posible evolución humana” es una necesidad no demandada. ¿Es acaso esto un dilema?

2) Quizá no haya que “educar para la vida”: Si la vida es un desastre, ¿por qué tanto énfasis en educar para la vida? Debemos educar para cambiarlo casi todo, y cambiarlo casi todo para mejorar la vida, incluyendo la muerte (HERRÁN; CORTINA, 2008). No sólo lo cortical o lo sonoro -la desaparición de la capa de ozono, el cambio climático, la galopante extinción de especies, etc.- ha de encender la alarma de la sensatez. Nuestro interior forma parte del paisaje, porque de hecho es su manantial y lo produce.

3) Quizá no se ha de pensar sólo en el niño o en el joven en primer plano: La infancia y la juventud componen la mayor parte de la población mundial. Aunque vayan a ser la sociedad de mañana, no constituyen causa de la actualidad. Al contrario: Los jóvenes son consecuencia de las sociedades adultas. Provienen de ellas y absorben de ellas lo mejor y lo peor por contagio y ejemplaridad. La problemática de los jóvenes no radica en ellos en primera instancia, sus raíces les traspasan y arraigan en el sector adulto. No acertaremos a educar a la sociedad futura si antes no empezamos por educarnos a nosotros mismos. En definitiva, educando al adulto educaremos a la infancia y a la juventud emergente. Pero, ¿qué entendemos por educar al adulto o a la sociedad? Fundamentalmente, incidir en aquellos sectores que más influyen en la adolescencia y la juventud. En educación el camino más corto no es la línea recta, sino la línea curva, y esta curvatura pasa por la familia y los medios de comunicación. Después, por los sistemas sociales que no se llaman escuela: familia, medios de comunicación, política, economía, religión... Se impone a mi juicio una reforma educativa de todos ellos para después proceder a una reforma educativa con todos ellos (HERRÁN, 1993, 2003, 2006). No se trata de pretender que la tribu-toda eduque (MARINA, 2004), sino de que la tribu entera antes se eduque, y que desde ese cultivo resulte la desidentificación de su tribuidad (o ego-tribal), para la reidentificación en la Humanidad, y aprenda a actuar no tanto "hacia” sí, sino “desde” sí misma, para la posible evolución humana. La diferencia preposicional resulta decisiva. Un sector adulto más culto comprenderá mejor al niño y al joven, pero también entenderá mejor la educación. M. Montessori hablaba de la “ceguera 
adulta”, entendía como “egoísmo inconsciente”. Éste se manifestaba desde que el niño nace y, específicamente, en el plano educativo. C. Freinet (1971, p.28) reparó en algo parecido:

4)

\begin{abstract}
A la mayor parte de los padres lo que les importa no es la formación, el enriquecimiento profundo de la personalidad de sus hijos, sino la instrucción suficiente para afrontar los exámenes, ocupar las plazas codiciadas, ingresar en tal escuela o meter el pie en alguna casa de negocios o en la administración del Estado.
\end{abstract}

Nosotros nos referimos a una “ceguera” mayor que incluye ésta: la que lleva al niño y al joven al adoctrinamiento en sus expectativas, sistemas de creencias, ismos, programas mentales colectivos, etc., e impide muchas veces que descubra desde su propio pensamiento.

\title{
5) Quizá la aceleración de los acontecimientos no sea la mayor preocupación
}

educativa: Estamos en los primeros milímetros de un viaje apasionante que acabamos de iniciar: la evolución humana. ¡Y ya experimentamos aceleración en acontecimientos, personas y sistemas! ¿Cómo puede ser posible? Desde una perspectiva social, también sentimos que perdemos el control. Parte de la sociedad comienza a deslizarse por la pendiente, y nos parece entrar en un tramo de aceleración y vértigo. Lo que hemos creado a veces se reconoce como atroz, porque se nutre de nosotros. Y nuestra adaptación es tan perversa que lo aceptamos como parte del pago, porque a los países ricos no suele afectar. Desde una perspectiva personal, a la sensación de aceleración podría contribuir la sospecha de que podemos salirnos de la vía, que quizá ya nos estamos saliendo, que la estabilidad del vehículo es escasa, que el estado del firme no es el adecuado o que nuestra pericia es escasa. A partir de aquí, se piensa que un incremento mayor de aceleración podría producir un accidente. Esta inferencia es, a mi juicio, errónea, porque la aceleración hace referencia a la variación de la velocidad en cada instante. No equivale a mayor velocidad, y menos a alta velocidad. Ahora bien, supongamos que en nuestro contexto la aceleración del vehículo social, gracias a las TIC, sí va unida a mayor velocidad de los hechos. Pues bien, aun así no nos parece que éste sea el problema principal del que debamos ocuparnos. El problema principal para nosotros, como pedagogos radica en dos aspectos:

a) El volante o la dirección: Ha de primar sobre el impulso o la desaceleración. La pregunta clave es: ¿hacia dónde vamos? o ¿para qué avanzamos? Tomar conciencia de ello puede ser bueno para evitar un percance, procediendo a desacelerar ${ }^{3}$, para controlar la

\footnotetext{
${ }^{3}$ Un ejemplo concreto es la aportación de C. Honore (2005), quien defiende la desaceleración en la vida cotidiana: comer, trabajar, comunicarse, sexualidad, etc.
} 
velocidad, y recuperar la dirección para volver a enfilar la carretera. Esto dependerá sobre todo de la formación del piloto, luego de su educación. M. Á. Santos Guerra (2008, p.3) reflexiona así:

Lo decisivo no es ir deprisa sino saber adónde se va. A veces damos la sensación de ser tripulantes afanados en alimentar la caldera del barco, extenuados por el trabajo pero despreocupados por el rumbo. ¿Y si fuéramos al abismo? ¿Y si estuviéramos dando vueltas en círculos concéntricos? No hay viento favorable para un barco que va a la deriva. No hay nada más absurdo que lanzarse con la mayor eficacia en la dirección equivocada.

b) La situación del centro de gravedad: Un centro de gravedad alto hace inestable a un vehículo a alta velocidad. Debe tomar las curvas con precaución y velocidad adecuada. Si no, podría volcar o salirse de la vía. La vida cotidiana y hasta nuestra educación exteriorizan a la persona, no satisfacen la necesidad de interiorización propia de todo ser humano. Vivimos una deslocalización interior. La cola se ha puesto delante y la cabeza se ha colocado detrás. Entre la aceleración exterior y la deficitaria desconexión esencial, el problema número 1 del ser humano es la segunda, no la primera. La situación es preocupante, porque los excesos de la primera minimizan la conciencia de la segunda.

En definitiva, la aceleración no es el principal objeto de preocupación educativa. El problema educativo básico es de orientación y estabilidad.

6) Quizá la respuesta educativa a la crisis social que atravesamos no pase por ofrecer sólo "nuevos aprendizajes del siglo XXI" a la infancia y a la juventud: Cuando se dice esto, podríamos estar equivocados, por dos razones:

a) Porque el aprendizaje como tal no siempre es formativo o deseable: "no todo aprendizaje significativo es positivo” (HERRÁN; GONZÁLEZ, 2002). El aprendizaje será formativo sólo si favorece la evolución de la persona desde una mayor complejidad de conciencia.

b) Porque para el siglo XXI hay varias clases de retos, incluidos los desafíos perennes. Por tanto, lo que es preciso atender son todos los fenómenos en su complejidad: futuros, contextuales, funcionales, perennes demandados y perennes no demandados (HERRÁN; CORTINA, 2008), superando la estrecha consideración de lo nuevo por lo actual. Desde otro punto de vista:

- Hay desafíos primarios, defensivos, para la supervivencia de entornos, contextos o situaciones. Son circunstanciales, actuales, inerciales, coyunturales, neutralizables. 
- $\quad$ Hay desafíos de segundo orden, para el desarrollo de la nueva sociedad emergente. Presentan características comunes para casi todos los entornos, asociadas al progreso. Requieren una reflexión de naturaleza anticipatoria. No son neutralizables, porque, una vez satisfechos, se abren a nuevos desafíos y emergencias de la misma naturaleza. Los denominamos futuros u orientados al futuro. F. Mayor Zaragoza (2000, p.21) ha concluido en el mismo sentido que nosotros:

El profesor debe atender de manera personalizada, porque cada ser humano es único. Pero la escuela tiene también otra función, la de vigía, de avizorar el futuro. Es necesaria una enseñanza anticipatoria, que prevea y se adelante a los acontecimientos.

Nosotros pensamos, en definitiva: anticipatoria sí, pero no nos refiramos sólo al cambio de la escuela de las aplicaciones disciplinares, de las nuevas tecnologías o de las lenguas extranjeras. Anticiparse es actualizar cuestiones perennes.

- Hay desafíos de tercer orden, para la posible evolución humana: Son independientes de contextos y sociedades, pero han de orientarse desde cada realidad. Presentan una naturaleza perenne, inherente a la condición humana. Partimos de la hipótesis enunciada por A. Rimbaud, el poeta que a los 20 años había escrito su obra literaria: "Es muy posible que nos hallemos en un tiempo en el que el futuro próximo hable la misma lengua del remoto pasado”. O sea, algo semejante a lo que expresaba E. Fromm (1987, p.45), cuando decía: "Puede decirse sin exageración que nunca estuvo tan difundido por el mundo como en la actualidad el conocimiento de las grandes ideas producidas por la especie humana, y que nunca esas ideas fueron menos efectivas que hoy”. El horizonte de los tiempos nuevos también debería asimilarse a cuestiones perennes, que también son actuales. En toda circunstancia pueden considerarse más recientes que los desafíos anteriores, porque son previos a ellos y se renovarán en su mismo sentido, y sin embargo aquellos son mudables. Entre los temas perennes podrían diferenciarse los demandados de los no demandados, casi siempre por una influencia directa del contexto ideológico. No están lo suficientemente actualizados.

Pues bien, lo normal es centrarse en los segundos, que son nortes para los primeros y comodines para los terceros. Nos inclinamos por un planteamiento equilibrado, con raíces y orientación evolutiva, centrada en la educación de la conciencia, que capacite a alumnos y profesores a la vez para dar respuestas constructivas y equilibradas a todos los retos que se representan. 
7) Quizá en la Educación en Valores no esté la solución: Los valores se prestan a la parcialización, falseamiento, distorsión, tergiversación y manipulación, por una deficiente definición del constructo. Los valores son ideales consensuados, bien nacionales, culturales, religiosos, sociales o personales. Por eso, en sentido estricto, no existen: existen cosas valoradas o a las que se adjudica un cierto valor. Y esta aplicación es casi siempre un invento arbitrario. Otras veces, la arbitrariedad consiste en apropiarse en exclusiva de aquella cualidad o abstracción comúnmente aceptada. Los valores son parte o formas del conocimiento. Denominamos ética al resultado de haber pensado bien. La verdadera ética no proviene de valores, no es exógena, no está asociada ni promovida por un ismo. El buen pensar tiene más que ver con la educación de la razón, que conduce a la observación, a la mirada, a la claridad o de la lucidez como consecuencia de cualidades o virtudes personales radicadas en el conocimiento que genera una conciencia capaz de ver más allá del ego.

Frente a ella, el pensamiento egocéntrico, inmaduro o parcial, se atribuye lo que a todos suena bien, para el propio beneficio. Esto ocurre también con los valores, inicialmente bienintencionados, condicionan y programan colectivamente. Desde esta perspectiva, los valores, cuya raíz etimológica es fuerza, traen como consecuencia la separación y el enfrentamiento (KRISHNAMURTI, 2008). En esta situación, la conciencia relativa no se libera del ego, y las valoraciones y sus fuentes acaban por ser promovidas por sistemas que precisan para su homeostasis de relaciones narcisistas, falta de humildad, dificultad de contemplarse desde fuera, ausencia de autocrítica, dificultad para rectificar, justificaciones y razones avaladas desde dentro, etc. Todo ello lastra la conciencia. Siendo así, el camino de la formación queda definido: Cuando se bota un globo, no sólo es necesario atender la seguridad de la barquilla y revisar la fuente de calor: es preciso soltar amarras y lastres. Para perder esta clase de afianzamientos es preciso aprender a dudar, luego a pensar; aprender a ser humildes, luego a autocriticarse, aprender a ser flexibles, luego a abrirse a otras posibilidades; aprender a cooperar luego a converger. En definitiva, aprender a superar dualidades, luego a razonar en clave de síntesis y a desarrollar un sentimiento de unidad más allá de los valores.

En la "sociedad del egocentrismo" que componemos aprendemos a incorporar, pero reducimos o eliminamos poco. Quizá los materiales de desecho de la conciencia (prejuicios, identificaciones dependientes, parcialidad en el sentir y en el razonar, rentabilidad sin plenitud, fanatismos, odios...) son esenciales para el afianzamiento de los valores egocéntricos anteriores a que nos referimos. Esto probaría la tesis con que arrancamos el parágrafo: "Los valores se prestan a su parcialización, falseamiento, distorsión [...]” (KRISHNAMURTI, 2008, p.123). Desde nuestra perspectiva, este fenómeno no se reconoce porque su eliminación 
sería costosísima, porque no hay alternativa -la historia demuestra que siempre son parcialesy porque no se desea hacerlo. Contradiría, además, el metavalor del "tener” y de los “personajes” (BLAY, 2008) que creemos ser. Otra razón es que miedo: a perder, a cambiar, a la muerte, aunque sea parcial. Y he aquí que éstos son otros polos del fracaso de la educación social. La persona orientada al crecimiento interior, a la autorrealización, a la síntesis entre la mediocridad y la conciencia es la excepción. Por tanto, como dice el proverbio japonés, el problema no es tanto que el pozo sea demasiado hondo, es que la cuerda es demasiado corta.

Otro método de diferenciación entre valores egocéntricos y valores de universalidad o educativos se basa en la respuesta a dos preguntas: “¿Valor para quién y para qué?” y “¿Valor en qué nivel de complejidad evolutiva?”. Esta característica no la dan los valores en sí, sino el uso que de los valores se hace. Por ejemplo, ¿¿son valores formativos la solidaridad, la empatía, la creatividad, la motivación, la autoestima, la autoevaluación, etc.? No puede haber respuesta positiva a priori: su bondad será relativa, y dependerá de su orientación, no de su intensidad. En efecto, todos ellos son esenciales, a la vez, para la destrucción y el dominio del otro, y para practicar el bien. Dependerá de si se desarrollan desde el ego o la conciencia, desde pensamiento egocéntrico o maduro. La segunda de las preguntas es la clave de las otras dos, y en ella nos centramos. Podríamos reconocer tres y sólo tres clases de acciones, proyectos, expresiones creativas, respuestas o valores, según su nivel de complejidad: 1) Acción para el logro. 2) Logro para mi sistema. 3) Mi sistema para la posible evolución humana. Cada uno de estos niveles supera lógicamente al anterior, porque lo abarca, como el producto engloba a la adición. Cada nivel implica formas más capaces de sentimiento y razón. Si por valor entendemos una consecuencia de haber pensado bien, inferiremos que situando el sentimiento, el pensamiento y la acción en el nivel 3 de complejidad de conciencia antes apuntado los valores así mismo se corregirían espontáneamente. En síntesis, proponemos tres métodos o caminos complementarios para avanzar en la formación en valores de un modo fiable:

- El "camino directo” del conocimiento desde una lógica dialéctica: Si la ética resulta del buen pensar, y ese razonar bien tiene algo que ver con la fiabilidad y la belleza ordenada de las cosas y de los fenómenos, quizá la lógica, comprendida como “método con que la razón ejerce su función” (GONZÁLEZ JIMÉNEZ, 2008, p.19) pueda comprenderse como la forma más elegante de la ética. Pero esa lógica no podrá ser dual, sino dialéctica, basada en el uso de la duda. El conocimiento tiene una naturaleza “dudosa” (RUSSELL, 1992), y es ésta característica la que le proporciona dinamicidad, incompleción y falibilidad. Si no hay duda, 
el conocimiento es una fotografía o un cadáver de la realidad, un asiento de su potencialidad sin actividad real. Podemos llamarlo "continente" o "contenido", es lo de menos. La duda tiene como método la complejidad para la síntesis, y como anhelo la adquisición de más conciencia. Allí donde ambas coinciden, o sea, en síntesis superadoras, se es consciente de la limitación de los componentes (tesis y antítesis relativas). Este método de la complementariedad indagadora es un medio honesto, apoyado en la realidad exterior y en la potencialidad sináptica o neurológica, válido para avanzar de la mano segura de la lógica dialéctica uno-ternaria (cucu). El resultado devuelve así a la naturaleza una belleza acrecentada.

- El "camino indirecto", centrado en las virtudes humanas: Por la labilidad del término “valor”, proponemos un cambio en la consideración de la Educación en Valores a favor de una Educación en Virtudes, como opción más fiable, por razones de peso: La Educación en Valores se apoya en una lectura social, tribal, intersubjetiva, sesgable y en último término arbitraria que la descalifica. La Educación en Virtudes se apoyaría en cualidades de la persona, y por tanto en una concepción más concreta, objetivable y generalizable. Al radicar en la persona individual, pueden ser más fácilmente vinculables a la formación y por ello incluyen una mayor alícuota de educatividad. Y es esta relación con la formación la que le suscribe, para nosotros, su metavalor definitivo. Si como decía Chauchard (apud MIRET MAGDALENA, 2004): “La ética es la continuación o prolongación de las virtudes humanas”, la Educación de la Conciencia, a que apuntamos, puede ser un camino excelente para emprender la Educación de la Razón, que no es diferente de la Educación de las Virtudes, desde una perspectiva formativa, laica y permanentemente evolutiva. Aun así, hay puntos de confluencia entre valores y virtudes, y ésas son articulaciones que pueden resultar fiables. Pero nótese que son los casos de virtudes asumidas como valores. Por ejemplo, la virtud-valor de la sensibilidad o de la responsabilidad. Desde el plano de la comunicación didáctica, proponemos trabajar preferentemente desde las virtudes que los alumnos valoran en lo concreto, relacionando con ello los objetivos personales para el bien del colectivo, como proponía Makarenko. Un enfoque didáctico como éste requiere por parte docente de gran dosis de observación y escucha, hasta el punto de tomar al niño como maestro de muchas virtudes naturales. En definitiva, responderemos afirmativamente a la pregunta de Séneca: “¿Es posible enseñar la virtud?”. Además, coincidimos con José Martí: “Tengo fe en el mejoramiento humano, en la vida futura, en la utilidad de la virtud”.

- El "camino en negativo" centrado en los lastres para la complejidad de la conciencia: Se trata de centrarse en los lastres que condicionan o escoran la evolución en 
complejidad de la conciencia. Desde esta perspectiva, el problema de los valores, que mejor mirado es el de las virtudes humanas, podría entenderse como un asunto inversamente dependiente del egocentrismo (epicentro de la inmadurez), de modo que bastará con reducir o disolver el "ego" o sus infantilismos en personas y colectivos para ganar espontánea, automáticamente en virtudes o valores deseables. Aunque Manuel Machado escribió: “No tener vicios no añade nada a la virtud”, creemos que al ser humano lo que le ocurre es justo lo contrario: el trabajo personal y social centrado en la pérdida de lastres, el desprendimiento, descascarillamiento o "muerte del ego" traerá automáticamente ganancia en altura de conciencia, mayor lucidez y mejor visión, y con ello virtudes personales y sociales formativas.

8) Quizá debamos redefinir el concepto de educación: O sea, proponer un cambio en su concepción para desembocar en un proyecto más complejo y ambicioso. Consideremos este encadenamiento: Educación es conocimiento y comunicación. Conocimiento y comunicación es construcción desde dentro. Construcción desde dentro es autoorientación. Autoorientación es autoeducación. Autoeducación es formación. Formación es madurez de la persona. Madurez de la persona es responsabilidad y compromiso para la mejora social. Responsabilidad y compromiso para la mejora social es evolución personal. Evolución personal es reducción de egocentrismo (individual y colectivo). Reducción de egocentrismo (individual y colectivo) es incremento de conciencia. Incremento de conciencia es capacidad de visión que da el conocimiento. Capacidad de visión que da el conocimiento es experiencia y transformación. Experiencia y transformación es posibilidad de autoconciencia. Autoconciencia es sentirse partícipes de un proceso cooperativo cuya meta común es la posible evolución humana.

Hoy la educación es pensada por otros sistemas fácticos, de modo que se pertenece poco a sí misma. Estamos muy lejos de todo lo anterior, pero esa misma lejanía quizá defina el contorno del horizonte formativo por venir. La educación es "energía primera de la humanidad” (BOUSQUET, 1974, p. 183) y origen de todo el bien en el mundo (KANT, 1983). Es útil no sólo para crecer, sino para repararse. Su fin último es la evolución humana o complejidad de conciencia generalizada. Requiere superar el confesionalismo y el laicismo para alcanzar el evolucionismo, al que se llega si, además de desarraigar las doctrinas religiosas de la educación pública, se hace lo propio con los nacionalismos (centrífugos y centrípetos), los etnocentrismos y en general todas las identificaciones organizadas incapaces de evitar la inducción de egocentrismos y narcisismos colectivos, doctrinas y sesgos a través 
de la enseñanza. Así pues, requiere aprender a interpretar la parcialidad definitoria de los ismos, así como el sentido y el modo de superarla, por la desidentificación y la convergencia que trae el conocimiento.

\section{VALUES IN A ADOLESCENT SOCIETY? EDUCATION OF THE CONSCIENCE}

ABSTRACT : We crossed a general crisis that some authors associate to a generalized social immaturity. In that context educative challenges as a result of redefining perceptions normally admitted are deduced.

KEYWORDS: Social immaturity. Egocentrism. Education of the conscience.

\section{REFERENCIAS}

BLAY, A. Ser: curso de Psicología de la autorrealización. Barcelona: Índigo, 2008.

BOUSQUET, J. La problemática de las reformas educativas. Madrid: Servicio de Publicaciones del Ministerio de Educación y Ciencia, 1974.

FREINET, C. Técnicas Freinet de la escuela moderna. 2.ed. México, DF: Siglo XXI, 1971.

FROMM, E. Sobre la desobediencia y otros ensayos. 2.ed. Barcelona: Paidós Ibérica, 1987.

GONZÁLEZ JIMÉNEZ, F. E. Qué es y de qué se ocupa la didáctica: sus fundamentos y métodos. In: HERRÁN GASCÓN, A. de la.; PAREDES, J. (Coord.). Didáctica general: la práctica de la enseñanza en educación infantil, primaria y secundaria. Madrid: Mc-Graw-Hill, 2008. p. 7-19.

HERRÁN, A. de la. Hacia una educación para la universalidad: más allá de los ismos. In: VALLE, J. M. (Dir.). Seminario de la identidad local a la ciudadanía universal: el gran reto de la educación contemporánea. Madrid: Fundación para la Libertad, 2008. p.209258.

Seis retos éticos para una reforma educativa con los medios de comunicación.

Étic@net, Granada, n.5, 2006. Disponible en:

<http://www.ugr.es/ sevimeco/revistaeticanet/index.htm>. Acceso em: 12 marzo 2007.

El siglo de la educación: formación evolucionista para el cambio social. Huelva: Hergué, 2003.

El ego humano: del yo existencial al ser esencial. Madrid: San Pablo, 1997. 
La educación del siglo XXI: cambio y evolución humana. Madrid: Ciencia 3, 1993.

HERRÁN, A. de la la; CORTINA, M. La muerte y su didáctica en educación infantil, primaria y secundaria. 2.ed. Madrid: Universitas, 2008.

HERRÁN, A. de la; GONZÁLEZ, I. El ego docente, punto ciego de la enseñanza, el desarrollo profesional y la formación del profesorado. Madrid: Universitas, 2002.

HONORE, C. Elogio de la lentitud. Barcelona: RBA Libros, 2005.

KANT, I. Pedagogía. Madrid: Akal, 1983.

KRISHNAMURTI, J. Aprender es vivir: cartas a las escuelas. Madrid: Gaia, 2008.

MARINA, J. A. Aprender a vivir. Barcelona: Ariel, 2004.

MAYOR ZARAGOZA, F. Educar para la individualidad: una formación permanente y de calidad para el siglo XXI. Acade, Madrid, v.28, p.18-22, 2000.

MIRET MAGDALENA, E. Nuevos horizontes en la sociabilidad humana: claves para la esperanza. In: CANTERAS MURILLO, A. (Coord.). Los jóvenes en un mundo en transformación: nuevos horizontes en la sociabilidad humana. INJUVE: Madrid, 2004. p.271-282.

MORIN, E. El método II: la vida de la vida. Madrid: Cátedra, 1983.

ROJAS, E. ¿Quién eres?: de la personalidad a la autoestima. Madrid: Booket, 2004. . Indicadores de madurez de la Personalidad. Cuadernos Empresa y Humanismo, Barcelona, v.23, p.1-13, 1990.

RUSSELL, B. El conocimiento humano. Barcelona: Planeta-De Agostini, 1992.

SANTOS GUERRA, M. Á. Carta abierta a mi Delegado Provincial de Educación. Escuela, Madrid, n.3798, p.3, 2008.

\section{BIBLIOGRAFIA CONSULTADA}

HERRÁN, A. de la; VILLENA, J. L. Integración curricular de la televisión. In: ORTEGA CARRILLO, J. A.; CHACÓN MEDINA, A. (Coord.). Nuevas tecnologías para la educación en la era digital. Madrid: Pirámide, 2006. p. 193-205. 